\title{
Articulating Complex Information Needs Using Query Templates
}

\author{
Olga Vechtomova ${ }^{1}$ and Hao Zhang \\ Department of Management Sciences, University of Waterloo
}

\begin{abstract}
In this paper we investigate the effectiveness of topic-independent query templates as a tool for assisting users in articulating their information needs. We hypothesize that topic-independent query templates can help users with complex information needs to express their requirements more accurately and in greater detail. We developed a set of query templates representing general semantic relationships between concepts, such as cause-effect and problem-solution. Each template was written in the form of a fill-in-the-blanks question. A user study was performed comparing the templatebased interface with a single-textbox search interface. Results demonstrate that, while users found the template-based query formulation less easy to use, the queries written using templates performed better than the queries written using the control interface with one query textbox.
\end{abstract}

Keywords: Query formulation, query templates, complex information needs, Rhetorical Structure Theory, interactive information retrieval, user study.

\section{Introduction}

While many users have relatively simple or general information needs, users who are familiar with a certain topic may have more specific or complex information needs. Having already some knowledge of a subject and its concepts or entities, such users may want to find information on a specific aspect of a certain entity, such as its cause, effect, how it can be prevented, what can be done to achieve it, or a relationship between entities, e.g., how does entity $\mathrm{X}$

\footnotetext{
${ }^{1}$ Correspondence to: Olga Vechtomova, Department of Management Sciences, University of Waterloo, 200 University Avenue West, Waterloo, ON, N2L 3G1, Canada, ovechtom@engmail.uwaterloo.ca.
} 


\section{Olga Vechtomova and Hao Zhang}

influence entity $\mathrm{Y}$ or what do entities $\mathrm{X}$ and $\mathrm{Y}$ have in common. We refer to such information needs as complex needs. Consider the following scenario: a business analyst wants to find information about the effects of credit card fraud on the U.S. banks. She has been working in banking and finance for a long time, therefore, she is not interested in documents explaining the banking system or types of credit card fraud. Instead, she would like to learn about the financial losses that banks incur due to credit card fraud and whether a growing number of fraud cases and banks' insufficient fraud prevention programs cause customers to leave.

In contrast, users unfamiliar with a subject have limited or no knowledge of its concepts and entities, and hence are less likely to have a clear understanding of what they need to know in this subject area. Their information needs are, therefore, likely to be relatively simple: to find information about the subject, so that they can learn more about it. An example scenario is a first-year university student beginning to work on a coursework paper about credit card fraud. He has no previous knowledge of this subject and does not have a clear idea what he needs to know. So, first he would like to read basic information about the subject.

In order to resolve complex information needs, an IR system, should, firstly, help users express different semantic relations between the concepts/entities of their interest. Secondly, it should have a retrieval model that would rank documents based on the likelihood that they contain the sought semantic relations between entities.

In this paper we focus on the first problem: helping users articulate complex information needs by expressing specific semantic relations between the entities of their interest. We propose a novel approach to query formulation, whereby the system suggests to the user a list of templates in the form of fill-in-the-blanks questions. Each of the templates expresses a topic-independent semantic relation, such as cause-effect and problem-solution. The user is invited to fill in as many or as few templates as they feel necessary to express their information need. An example of a template is "What effect does have on ?’

We implemented two query formulation interfaces: a template-based interface and a single-textbox interface, and conducted a user study to learn how effectively users formulate queries with them. It is reasonable to expect that a template-based query formulation interface may be more effective as a front-end to an IR system that makes use of semantic relations in document ranking, as opposed to a bag-of-words IR system. However, no such system is presently available, and the developed interfaces were evaluated as front-ends to one of the state-of-the-art IR systems, Okapi [1]. The goal of the user study was to investigate whether users can be expected to effectively articulate complex information needs by filling in topic-independent templates. Two major research questions we explored are:

RQ1: Are users able to articulate complex information needs using topic-independent query templates?

RQ2: When used with a bag-of-words retrieval system, do the queries formed using template-based interface lead to better search results than queries formed using a single-textbox interface?

The rest of the paper is organized as follows: Section 2 discusses related work, Section 3 describes the construction of query templates, Section 4 reports the experiment design and protocol, the results of the experiments are analysed in Section 5, Section 6 provides a qualitative analysis of several user queries, finally Section 7 concludes the paper and outlines future research directions.

\section{Related work}

One of the central problems in the information retrieval process is the gap between a person's information need and a query statement that she submits to an IR system. It is usually difficult for people to express their information 


\section{Olga Vechtomova and Hao Zhang}

needs informally using a natural language, let alone to formulate them as effective queries for an IR system. Belkin et al. [2] in their well-known ASK (Anomalous State of Knowledge) hypothesis stated that "an information need arises from a recognized anomaly in the user's state of knowledge concerning some topic or situation and that, in general, the user is unable to specify precisely what is needed to resolve that anomaly." [2] (p. 62). Most interfaces to experimental and operational IR systems are developed based on the assumption that it is difficult or impossible for users to express their information needs precisely. Thus, for example, most commercial search engine interfaces have small query textboxes that invite short queries. On the other hand, experiments using test collections, such as TREC, showed that query length has a positive effect on performance [3]; therefore it is worth encouraging users to submit more detailed queries.

While in operational IR settings, the average query length is 2-3 words [4], a number of user studies showed that users are willing to submit longer and more expressive queries when encouraged to do so [5,6]. In a user study by Belkin et al. [5], an experimental interface, which encouraged users to submit a longer query by providing them with a larger query textbox, was compared to a standard interface. The study proved that users submitted longer queries when using the experimental interface, and that reported user satisfaction was higher for the experimental interface than the control interface. However, no correlation between query length and performance was observed.

Kelly et al. [6] conducted an experiment within the framework of the HARD (High Accuracy Retrieval from Documents) track of TREC 2004. The HARD track protocol included a one-time interaction with the users (NIST assessors), whereby upon receiving an initial query statement, participating sites could send to the user a clarification form, which could ask for any information about the user's information need. In their clarification form Kelly et al. asked the user to supply information in response to the following questions/prompts: "Describe what you already know about the topic."; "Why do you want to know about this topic?" and "Please input any additional keywords that describe your topic." These prompts were followed by large textboxes, inviting long answers. The questions/prompts were intended to encourage the user to talk more about his/her information need. The results point to a relationship between query length and performance, and indicate that user-supplied responses improved retrieval performance compared to their initial queries. The systems in the above studies encourage and prompt users to talk more about their information needs, but they do little to help them articulate the relationships between concepts and entities that are important to their information needs.

Price et al. [7] proposed the semantic components model, which is intended to represent document contents in terms of various domain-specific topic aspects. Examples of semantic components in the medical domain are etiology and treatment. They developed and evaluated an experimental interface for a medical domain search system. Users were invited to enter their query plus fill in any textboxes representing various domain-specific semantic components. Documents in their collection were annotated with the semantic components, and an IR system ranked documents based on the match between the user-specified semantic components, and the semantic components assigned to documents. They reported improved performance compared to the control system, which did not use semantic components in query formulation and document ranking.

One of the contributions of our research is investigation of the template-based query formulation under domainindependent search conditions. The results of the user study suggest that while users found the process of templatebased query formulation not as easy as using single-textbox interface, the queries they built tended to enhance the retrieval performance of a bag-of-words IR system.

The problem of satisfying complex information needs is relatively unexplored, but is beginning to gain attention. Most of the experimental research was conducted over the last few years within the framework of three TREC initiatives: the Relationship task of the Question Answering 2005 track and Complex Interactive Question Answering (CIQA) 2006 and 2007 tasks [8]. One of the aims of CIQA was also to study the interactive aspect of the IR process within the context of the complex question answering task. The opinion retrieval task in the Blog track [9] of TREC 


\section{Olga Vechtomova and Hao Zhang}

focused on one type of complex information needs - finding documents containing positive and negative opinions about sought entities.

Over the last few years, active research has been conducted in the area of automatic identification and classification of semantic relations. Various evaluation frameworks, such as SemEval [10] and Recognizing Textual Entailment (RTE) task [11] in the Text Analysis Conference (TAC), promote the development of new techniques, which may lead to the development of IR systems that make use of semantic relations between entities in document ranking. Therefore, it is important to investigate how users can communicate to an IR system the semantic relations between entities that they are interested in. Some recent work in the field of semantic relation identification and classification is summarized below.

Hearst [12] proposed the idea of mining a large corpus for lexical patterns that would help to identify a specific relation (hyponym-hypernym). Turney and Littman [13] classify noun-modifier pairs according to the semantic relations between them. They use counts of various short joining terms, obtained by web queries to measure the similarity between the test pair and the training pairs. Nakov and Hearst [14] propose a method that mines the Web to build a set of features that frequently occur in the same sentences as the target word pair. They then calculate similarity using Dice coefficient between the feature vector representing the test example with the vectors representing the training examples. Beamer et al. [15] used syntactic, lexical and semantic features from various knowledge bases. For example, they used information from WordNet and other sources to determine whether a noun belongs to spatial or temporal category. This served to recognize near-miss examples in cause-effect relationships, such as "activation by summer". Within the area of opinion detection, Hurst and Nigam [16] propose a method of identifying sentences that are relevant to some topic and express opinion on it. Yi et al. [17] propose to extract positive and negative opinions about specific features of a topic. By feature terms they mean terms that have either part-of or attribute-of relationships with the given topic or with a known feature of the topic. Yang et al. [18] relied on the opinion terms, rare non-dictionary terms, and phrases involving "I" or "you" pronouns compiled semiautomatically from a training dataset.

\section{Constructing query templates}

In order to identify a set of possible query templates, we have manually analyzed the descriptions and narratives of 100 TREC topics (\#301-400) from TREC 6 and 7 ad-hoc tracks. The goal of this analysis was to identify a set of domain- and topic-independent semantic relations between concepts, about which users want to find information. We used the Rhetorical Structure Theory [19] to guide us in the analysis of the TREC topic narratives.

Rhetorical Structure Theory (RST) is a framework for the description and analysis of texts in terms of functions and roles played by different segments of text. Each natural language text has some coherence or unity, which means that every segment, such as a clause or sentence, has a certain role or function in text. Consider the following sentence: “Jack couldn't get on the plane. He lost his ticket.” In this example, the first sentence represents an effect, while the second one represents a cause. Compare the previous example with the following: "Jack couldn't get on the plane. He likes apples." These two sentences lack coherence because it is not clear to the reader why they are placed together.

The RST analysis consists of identifying coherence relations between the segments of text, i.e. the role that one segment plays in relation to the other. In most cases, one of the segments is more essential to the overall communicative goal of the text, and is called nucleus, while the other, less essential, called satellite. In the first example above, the relation between segments is “Cause”. Here, the effect (that Jack couldn't get on the plane) is 


\section{Olga Vechtomova and Hao Zhang}

more important than the cause (that he lost his ticket), therefore, the effect is the nucleus and the cause is satellite. Such RST relations are called nucleus-satellite relations. Another kind of relations is multinuclear, in which neither of the relations is more central to the communicative goal of the text. An example of such relation is "Contrast", whereby two nuclei are compared and contrasted.

The nucleus-satellite relations can be of two types: presentational and subject-matter. "Presentational relations are those whose intended effect is to increase some inclination in the reader, such as the desire to act or the degree of positive regard for, belief in, or acceptance of the nucleus. Subject matter relations are those whose intended effect is that the reader recognizes the relation in question" [20]. An example of a presentational relation is "Evidence", which means that a reader's understanding of the satellite increases his/her belief/acceptance of the nucleus. Our focus was on identifying subject-matter relations between concepts in the text of TREC topic descriptions and narratives. The RST subject-matter relationships include: Circumstance, Condition, Elaboration, Evaluation, Interpretation, Means, Non-volitional Cause, Non-volitional Result, Otherwise, Purpose, Solutionhood, Unconditional, Unless, Volitional Cause, Volitional Result.

The analysis of the TREC topics was done by one of the authors of the paper. After reading the text of the description and narrative for a topic, the text was annotated with the RST subject-matter relations. The number of instances of each relation was recorded. We dropped four infrequent relations: "Circumstance", "Condition", "Unconditional" and "Unless", and added "Contrast" multinuclear relation. For each of these relations we then wrote one to two query templates in the form of fill-in-the-blanks questions. The phrasing of the templates was inspired by the actual questions that TREC topic authors used in the topic descriptions and narratives.

Table 1. Question templates and corresponding RST relations. $\mathrm{N}_{\text {completed }}-$ the number of users who completed each template in our user study.

\begin{tabular}{|c|c|c|}
\hline RST relation & Template & $\mathbf{N}_{\text {completed }}$ \\
\hline Elaboration & Find cases/instances of & 28 \\
\hline Elaboration & Find statistical data on & 16 \\
\hline $\begin{array}{l}\text { Cause (volitional/ } \\
\text { non-volitional) }\end{array}$ & What causes __? & 21 \\
\hline $\begin{array}{l}\text { Result } \\
\text { (volitional/ } \\
\text { non-volitional) }\end{array}$ & What effect does ___ have on ___ ? & 17 \\
\hline Purpose & What are existing/potential uses/applications of & 23 \\
\hline Purpose & What is the goal/purpose of & 15 \\
\hline Otherwise & What impedes (gets in the way of) & 17 \\
\hline Otherwise & $\begin{array}{l}\text { What has been done (could be done) to alleviate/reduce the effect of } \\
\text { on }\end{array}$ & 20 \\
\hline Means & $\begin{array}{l}\text { What has been done (could be done) to increase the effectiveness/effect } \\
\text { of __ on ? }\end{array}$ & 18 \\
\hline Evaluation & think/ say about & 17 \\
\hline Evaluation & Find positive and/or negative aspects (pros/cons) of & 18 \\
\hline Solutionhood & What methods/procedures are (can be) used to & 15 \\
\hline Contrast & Compare _ and _ (in terms of __ $)$. & 7 \\
\hline
\end{tabular}

In total, 13 query templates representing general semantic relations between concepts were written as a result of our analysis. The templates and the corresponding RST relations are presented in Table 1. As can be seen from the 


\section{Olga Vechtomova and Hao Zhang}

table, there is no one-to-one mapping between the RST relations and the developed templates. For example, Nonvolitional Cause [Nucleus (N) is caused by Satellite $(\mathrm{S})$, where S is a non-volitional action] and Volitional Cause [N is caused by $\mathrm{S}$, where $\mathrm{S}$ is a volitional action] relations are represented by one template "What causes__ ?" Two templates represent the relation Otherwise [realization of $\mathrm{N}$ prevents realization of S]: "What has been done (could be done) to combat/alleviate the effect of ___? ? and “What impedes (gets in the way of) ___? The users are expected to interact with the templates by completing those they find relevant to their information needs.

We realise that the RST relations and the fill-in-the-blanks questions, into which they were cast, do not represent all possible entity relations about which users may want to find information. This set of relations, however, is broad enough to investigate the research questions posed by our study.

\section{User study}

\subsection{Experiment Design}

The goal of the user study is two-fold: (1) to investigate whether users are able to articulate complex information needs using query templates and (2) to determine how effective the template-based queries are when used with a bagof-words IR system.

To investigate these questions, the following types of data were gathered in the course of the user study:

- Retrieval performance values based on the users' binary relevance judgments (Precision at 5, 10 and 15 documents retrieved in response to the users' queries);

- Search session logs (e.g., time to formulate the query, time to read/judge documents, number of query terms entered, number of templates filled in);

- The users' subjective comments (e.g., satisfaction with the search results, satisfaction with the overall system, familiarity with the topic prior to search) elicited via a questionnaire.

The data gathered via search session logs was used to measure the users' ability to formulate queries using templates, while the retrieval performance values and users' reported satisfaction levels were used to measure the retrieval effectiveness of template-based query formulation.

Two search interfaces were developed: the control and the experimental (template-based). The control interface contained one large textbox, where the user was invited to enter any words, phrases and sentences representing his/her information need (Figure 1). The textbox of the control interface was deliberately made large to encourage entry of long queries. The experimental interface consisted of 13 topic-independent query templates (Figure 2). The retrieval system used with both interfaces was Okapi with the default b and k1 values [21].

Thirty users, who are graduate students at the University of Waterloo, participated in the study. The user demographic details elicited through a short entry questionnaire are summarised in Table 2. As the search tasks, we used 10 topics from the TREC HARD 2004 track [22] collection. The topics are not the same that were used to create the templates using RST analysis. The first criterion for the selection of topics was that they represent a complex information need, which was taken to mean a case where the topic narrative contains at least one general semantic relation. The second criterion was that topics had 10 or more relevant documents in the HARD 2004 collection. This 


\section{Olga Vechtomova and Hao Zhang}

was done to eliminate topics with insufficient coverage in the collection. An example of the selected topic is given in Table 3. The collection on which the search tasks were performed was HARD 2004, consisting of 635,650 documents (newswire articles).

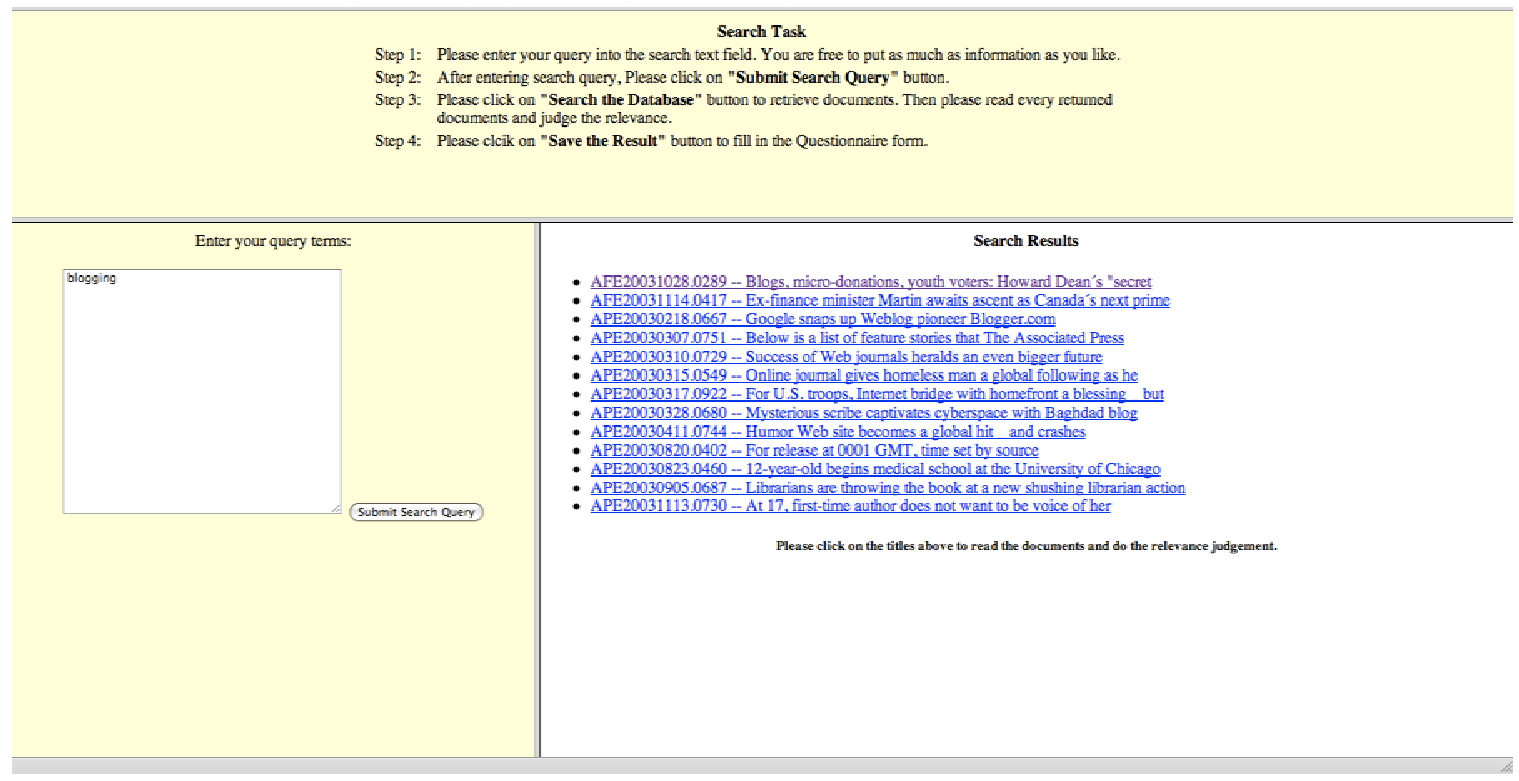

Figure 1. The control interface.

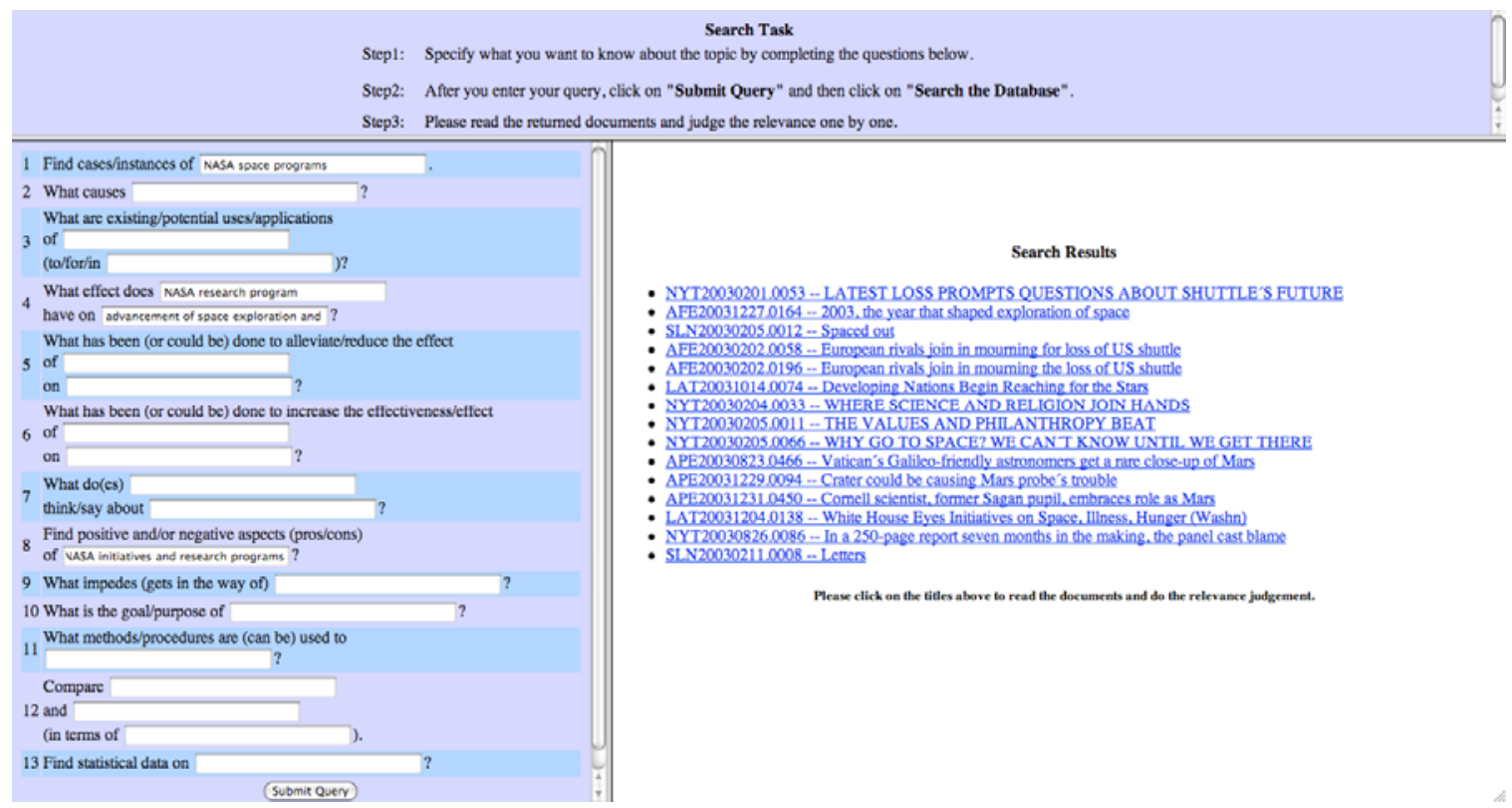

Figure 2. The experimental (template-based) interface. 


\section{Olga Vechtomova and Hao Zhang}

Table 2. User characteristics

\begin{tabular}{|l|r|}
\hline Question & Number of responses \\
\hline Age group & 0 \\
\hline 19 and under & 25 \\
\hline $20-29$ & 4 \\
\hline $30-39$ & 1 \\
\hline $40-49$ & 0 \\
\hline $50-59$ & 21 \\
\hline Gender & 9 \\
\hline Male & \\
\hline Female & 27 \\
\hline How often do you use search engines? & 3 \\
\hline Every day & 0 \\
\hline Several times per week & 0 \\
\hline Less than once a week & 3 \\
\hline Very rarely & 1 \\
\hline What do you use search engines for? & 26 \\
\hline Work/Study & 16 \\
\hline Personal & 14 \\
\hline Both & \multicolumn{2}{|r|}{} \\
\hline Do you use advanced search engine functions? & \\
\hline Yes & \\
\hline No & \\
\hline
\end{tabular}

Table 3. Example of the selected TREC topic

Number: 406

Title: The diamond Industry

Description: What levels of corruption exist in the Diamond Industry and how does the development of a synthetic flawless diamond impact the industry?

Narrative: The diamond industry has long been associated with corruption on many levels. With the emergence of a new synthetic stone, the industry finds itself at a crossroads. Virtually anything dealing with corruption in the diamond industry and the effects generating from the development of the synthetic gem is on topic. Articles relating to the technical aspects of cutting and setting diamonds are off topic. Scientific articles are also off topic.

The experiment was designed using the Latin Square principle. Each user performed two search tasks, one with the control interface and one of the topics, and the other with the experimental interface and a different topic. The order of the systems was rotated. For example, two topics A and B were assigned to six users as shown in Table 4. The rationale for using TREC topics as search tasks, as opposed to letting the users think of their own information needs, is that by giving the same topic to 3 users with the control system, and 3 - with the experimental, we are able to control the effect of the topic on the retrieval performance. 


\section{Olga Vechtomova and Hao Zhang}

Table 4. Experiment design

\begin{tabular}{|c|c|c|c|c|}
\hline \multirow{2}{*}{ User \# } & \multicolumn{2}{|c|}{ Search task 1 } & \multicolumn{2}{c|}{ Search task 2 } \\
\cline { 2 - 5 } & Interface & Topic & Interface & Topic \\
\hline 1 & Control & A & Experimental & B \\
\hline 2 & Experimental & A & Control & B \\
\hline 3 & Control & B & Experimental & A \\
\hline 4 & Experimental & B & Control & A \\
\hline 5 & Control & A & Experimental & B \\
\hline 6 & Experimental & A & Control & B \\
\hline
\end{tabular}

\subsection{Experimental Protocol}

Upon arrival each participant read and signed the informed consent letter and was given a short entry questionnaire, followed by a brief tutorial of the first system (experimental or control). The participant was then shown the TREC title, description and narrative of the assigned topic. Then, he/she was presented with 3 relevant documents for this topic, which were randomly selected from HARD 2004 relevance judgments file. The relevant documents were shown to the participants in order to increase their level of familiarity with the topic, since we hypothesize that template-based interface would be helpful to expert users with complex information needs. Such users already have a high level of familiarity with the subject and may be interested in learning about specific relationships between concepts or entities in their domain of interest. On the contrary, somebody having little or no familiarity with the topic is unlikely to benefit from the use of query templates, as he may simply have insufficient knowledge to identify related concepts and formulate complex queries.

The user was then presented with the corresponding query formulation interface (experimental or control) and was asked to formulate a query based on the topic given to her. The text of the topic was not displayed on the screen at the query formulation stage (see Figures 1 and 2) to prevent copying and pasting, but was given to the user on paper. After the user submitted the query, the query terms were stemmed, and document retrieval was performed using Okapi BM25. The three relevant documents shown to the user earlier were excluded from the search results. We also excluded duplicate documents from the retrieved set. The top 15 retrieved documents were shown to the user, who then judged each of them as relevant or non-relevant. At the end of each search task the user was given a short questionnaire eliciting feedback regarding the system used for that task. After a short break the user proceeded to the second search task. The experimental protocol for the second search task was the same as for the first. The experiment received full ethics clearance from the Office of Research Ethics at the University of Waterloo.

\section{Results and discussion}

\subsection{Retrieval Performance}

The performance of the user queries formulated by means of the template-based and the control interfaces was measured using precision at 5, 10 and 15 documents. The results are summarized in Table 5. 


\section{Olga Vechtomova and Hao Zhang}

Table 5. Performance of the queries formed using the control and template-based interfaces $(*$ is statistically significant; t-test, $\mathrm{p}=0.037$ )

\begin{tabular}{|l|l|l|l|}
\hline Interface & P@5 & P@10 & P@15 \\
\hline Control & 0.5133 & 0.4767 & 0.4667 \\
\hline Template-based & 0.6467 & $0.6067^{*}$ & 0.5533 \\
\hline
\end{tabular}

As can be seen from the table, the template-based interface helped users to formulate on average better queries that led to higher performance in all measures. Performance of the template-based system measured in P@5 is 25.97\% higher than the control, in P@10 it is 27.27\% higher and in P@15 it is 18.57\% higher. The improvement in P@10 is statistically significant.

\subsection{Query and Search Session Characteristics}

We have logged different parameters of the user search sessions, namely: time spent reading the 3 sample relevant documents shown to users prior to each search session, time spent formulating the query, time spent reading and judging the relevance of the top 15 retrieved documents, number of query terms entered (tokens), number of unique query terms entered (types) excluding stopwords, and inverse document frequency (idf) weight of the query terms entered. Table 6 summarizes the average values of these parameters. As we can see, the users spent on average 27 seconds more formulating the query using the templates, compared to entering the query into one textbox in the control interface. This observation is not surprising, since the templates require longer time to read and think whether and how each fill-in-the-blanks question relates to the user's information need.

Table 6. Characteristics of the search sessions using control and template-based interfaces

\begin{tabular}{|l|l|l|}
\hline & \multicolumn{1}{|c|}{$\begin{array}{c}\text { Control } \\
\text { (mean } \pm \text { stdev) }\end{array}$} & $\begin{array}{c}\text { Template-based } \\
\text { (mean } \pm \text { stdev) }\end{array}$ \\
\hline Time spent reading 3 sample relevant documents (min:sec) & $09: 28 \pm 06: 10$ & $08: 18 \pm 05: 31$ \\
\hline Time spent formulating the query (min:sec) & $04: 08 \pm 03: 39$ & $04: 35 \pm 03: 36$ \\
\hline Time spent reading and judging 15 retrieved documents (min:sec) & $22: 28 \pm 14: 22$ & $22: 09 \pm 12: 28$ \\
\hline Number of all query terms (tokens) including stopwords & $16.17 \pm 9.9$ & $31.83 \pm 19.36$ \\
\hline Number of unique query terms (types) excluding stopwords & $10.2 \pm 5.68$ & $12.03 \pm 5.15$ \\
\hline idf of non-stopword query terms & $4.05 \pm 1.09$ & $3.96 \pm 0.78$ \\
\hline
\end{tabular}

An interesting parameter is the number of query terms entered. The users on average entered 16.17 query term tokens (including stopwords) using the control interface, and 31.83 using the template-based interface. The difference (96.91\%) is statistically significant (t-test, $\mathrm{p}=0.0002$ ). The number of query terms without duplicates and stopwords is 10.2 (control) and 12.03 (template-based). The difference (17.97\%) is not statistically significant. Interestingly, on average 19.8 query terms entered using the experimental interface are either duplicates of the words previously entered for the same query by the user, or stopwords. At the same time, in the control interface, there are only 5.97 such words on average. This is not entirely unexpected since the user may be interested in different aspects of the same concept and enter it into several templates. Nevertheless, the users entered on average more unique nonstopword query terms using the template-based interface, which may be one of the reasons for its higher retrieval performance, compared to the control interface. The average idf of query terms is slightly higher (4.4\%) for the control interface, but the difference is not statistically significant. 


\section{Olga Vechtomova and Hao Zhang}

The average number of templates filled in by the users is 7.8 (standard deviation 3.13), which suggests that users were able to interpret and understand the templates in the context of their assigned search task. The number of times each template was completed by users $\left(\mathrm{N}_{\text {completed }}\right)$ is given in Table 1 . The RST relations corresponding to the most frequently filled in templates are "Elaboration" and "Purpose". The relation corresponding to the least frequently filled in template is "Contrast".

Since in our experiment each of the TREC topics was assigned to three users using the template-based system, we calculated the agreement among the users with respect to the templates they filled in for the assigned topic. The Fleiss Kappa is 0.14 , which corresponds to slight agreement. The reason why no strong agreement was observed is probably due to some flexibility inherent in the assigned search tasks: while the users were given rather detailed topic descriptions, they were able to interpret them somewhat differently based on their previous knowledge of the subject, focusing on different aspects of the topic, and therefore, filling in different templates. In Section 6 we discuss some cases where the users filled in the templates differently for the same topic.

\subsection{User satisfaction}

After each search session each user was asked to complete a short exit questionnaire designed to elicit subjective feedback regarding the search session and the interface. The questionnaire had one open-ended question "Please describe difficulties (if any) in formulating the query" and six close-ended questions, each with four answers to choose from, for example:

Q6. How satisfied are you with the search results?

- Very satisfied. I found most of the information I was searching for.

- Somewhat satisfied. I found some relevant information.

- Not too satisfied. Most of the sought information was not found.

- Not satisfied at all. None of the sought information was found.

The responses to the questions were then mapped to a 4-point scale with 1 being the most negative response and 4 - the most positive. The average responses are given in Table 7.

Table 7. Responses to questions in the exit questionnaire (* is statistically significant; t-test, $\mathrm{p}=0.01$ )

\begin{tabular}{|l|l|l|}
\hline & \multicolumn{1}{|c|}{$\begin{array}{c}\text { Control } \\
\text { (mean } \pm \text { stdev) }\end{array}$} & $\begin{array}{c}\text { Template-based } \\
\text { (mean } \pm \text { stdev) }\end{array}$ \\
\hline Understanding of the description of the search task & $3.63 \pm 0.56$ & $3.47 \pm 0.63$ \\
\hline Familiarity with the search topic before the start of the search session & $2.53 \pm 1.04$ & $2.47 \pm 1.14$ \\
\hline Familiarity with the search topic after reading three related articles & $3.3 \pm 0.59$ & $3.13 \pm 0.63$ \\
\hline Easiness in formulating the query & $3.23 \pm 0.73$ & $2.87^{*} \pm 0.78$ \\
\hline Satisfaction with the search results & $2.77 \pm 0.9$ & $3.17 \pm 0.79$ \\
\hline Overall satisfaction with the search system & $2.73 \pm 0.87$ & $3.1 \pm 0.71$ \\
\hline
\end{tabular}

The users on average had similar levels of understanding of the descriptions of the search tasks (topics) given to them with the control (3.63) and template-based (3.47) interfaces. They also had similar average levels of familiarity with the assigned topics before the start of the search sessions using control (2.53) and template-based (2.47) interfaces. Showing them 3 sample relevant documents increased the average familiarity levels to 3.3 and 3.13 respectively. 


\section{Olga Vechtomova and Hao Zhang}

The users found the process of formulating queries using templates (2.87) not as easy as using the single textbox in the control interface (3.23). The difference is statistically significant. One of the users explained in his/her response to the open-ended question: "It is not easy to put words in the query templates to fit the things I have in my mind to query." Another user said: "The fixed patterns seem to add more difficulties in expressing the original meaning of the question". Yet, another user commented: "Having a single line of text instead of an entire text area felt restrictive. I also felt that the provided 'questions' weren't geared very well towards this topic, so I didn't use very many of them. However, I felt that the results returned by this search were much more relevant than in the previous condition.” Some users were concerned that they entered the same word into several templates, thus one of the users mentioned "trying to come up with new terms to avoid repetition” as one of the perceived difficulties.

The reported user satisfaction with the search results returned by the template-based system is on average $14.5 \%$ higher than that for the control system, and the overall satisfaction with the template-based search system is $13.41 \%$ higher than the satisfaction with the control system, although the differences are not statistically significant. One user said that the template-based system allowed him/her to "...express the type of information explicitly...”, at the same time what this user disliked is that his/her information need required more than one template to express it. Another user commented, "The 13 lines in the query templates pretty much cover all question one would have, but it takes time to match my questions to the write line. Overall I feel the query templates are reasonably useful”. Some users felt that the terms they entered depended on the templates, e.g., "I found that I tend to come up with my search terms based on the question part in query templates, instead of independently creating the search terms”.

\section{Discussion}

To achieve a better understanding of the users' interaction with the templates, we performed a qualitative analysis of several user sessions. Specifically, we analysed query formulation by the users who were assigned topic 443 (Table 8). As mentioned earlier in our experiment, the same topic was assigned to three users using the template-based system, and three users using the control system.

Table 8. Topic 443.

Number: 443

Title: Hand-Held Electronics

Description: New technologies in the areas of cell phones, PDAs, and so forth.

Narrative: As technology more and more focuses on adapting old technology for smaller scales, new advances are abounding in the consumer markets, including advances in cell phone technology, PDA technology, notepad PCs, and digital cameras. Advances in all types of hand-held technology are on topic. Reports on large-scale technology, unless substantially applicable to hand-held technology as well are off topic. In such a case, the article itself must make the cross-application to hand-held technology. 


\section{Olga Vechtomova and Hao Zhang}

Table 9. Filled in templates by user 1 for topic 443.

\begin{tabular}{|l|}
\hline 1. Find cases/instances of portable electronics. \\
\hline 3. What are existing/potential uses/applications of portability (to/for/in electronics)? \\
\hline 6. What has been (or could be) done to increase the effectiveness of miniaturization on electronics? \\
\hline 8. Find positive and/or negative aspects (pros/cons) of hand-held devices? \\
\hline 10. What is the goal/purpose of portability? \\
\hline 11. What methods/procedures are used (can be used) to do/achieve make devices smaller? \\
\hline 13. Find statistical data on portable electronics? \\
\hline
\end{tabular}

Table 10. Filled in templates by user 6 for topic 443.

\begin{tabular}{|l|}
\hline 1. Find cases/instances of mobile phone, PDA, digital cameras. \\
\hline 2. What causes advancement in technology? \\
\hline 4. What effect does internet have on mobile phone? \\
\hline 5. What has been (or could be) done to alleviate/reduce the effect of website compatibility on PDA? \\
\hline 8. Find positive and/or negative aspects (pros/cons) of digital vs. film? \\
\hline 9. What impedes (gets in the way of) trading with PDA? \\
\hline
\end{tabular}

Table 11. Filled in templates by user 21 for topic 443.

\begin{tabular}{|l|}
\hline 1. Find cases/instances of hand-held electronics. \\
\hline 2. What causes new hand-held technologies to succeed? \\
\hline 3. What are existing/potential uses/applications of smart phones (to/for/in __ \\
\hline 4. What effect does UMTS have on cell phone adaption rates? \\
\hline 5. What has been (or could be) done to alleviate/reduce the effect of location on poor reception? \\
\hline 6. What has been (or could be) done to increase the effectiveness of messaging on cellphones? \\
\hline 7. What do(es) RIM think about the blackberry? \\
\hline 8. Find positive and/or negative aspects (pros/cons) of advances in hand-held technology? \\
\hline 9. What impedes (gets in the way of) consumer awareness of new cellphone features? \\
\hline 10. What is the goal/purpose of PDA innovation? \\
\hline 11. What methods/procedures are used (can be used) to do/achieve link multiple wireless devices? \\
\hline 12. Compare iPhone and Blackberry in terms of \\
\hline 13. Find statistical data on smart phone adaption rates? \\
\hline
\end{tabular}

As can be seen from Tables 9, 10 and 11, the users were able to fill in many templates, with user 21 filling in all 13 templates. It is evident from this example that the templates prompted the users to think about different aspects of cell phone technology and industry, such as the effect of UMTS (Universal Mobile Telecommunications System) on cell phone adoption rates, methods of linking multiple wireless devices and comparison of iPhone and Blackberry. Interestingly, while the users entered very similar words and phrases in template 1: "portable electronics", "hand-held electronics" and "mobile phone, PDAs, digital cameras", some other templates were completed quite differently. For example, template 4 was completed as "What effect does internet have on mobile phone?" by user 6, and as "What 


\section{Olga Vechtomova and Hao Zhang}

effect does UMTS have on cell phone adaption rates?” by user 21. Another example is template 11: user 1 filled it in as "What methods/procedures are used (can be used) to do/achieve make devices smaller?", while user 21 completed it as "What methods/procedures are used (can be used) to do/achieve link multiple wireless devices?”. It is evident that the users filled in the templates based on their knowledge of and interest in different aspects of the topic. Such differences in interests explain the overall low agreement in filling in the templates for the same topic as mentioned in Section 5.2.

For comparison, Table 12 shows the queries formulated by users for the same topic using the control interface. It is worth mentioning that user 30 formulated the query in the form of complete sentences, which resembled the templates. This user interacted with the control interface in the second session, so it is possible that she/he was influenced by the template-based interface she/he interacted with in the first session. Note that in Tables 9-12 we retained the original text entered by the users, which includes some typographical errors.

Table 12. Queries entered by the users using the single textbox interface for topic 443.

\begin{tabular}{|l|l|}
\hline User 11 & blackberry "reseach in motion" RIM "cell phone" mobile \\
\hline User 16 & next generation mobile phone technology handheld microsoft-powered cell phone \\
\hline User 30 & $\begin{array}{l}\text { Compare the Blackberry, a PDA, and a cell phone. What are the advantages and disadvantages of } \\
\text { each? What are the market share of each in the U.S. and the U.K. and Germany? }\end{array}$ \\
\hline
\end{tabular}

Table 13. Performance of the queries formulated using the template-based and control interfaces for topic 443.

\begin{tabular}{|l|l|l|l|l|l|l|l|}
\hline \multicolumn{4}{|c|}{ Experimental system } & \multicolumn{4}{c|}{ Control system } \\
\hline User & P@5 & P@10 & P@15 & User & P@5 & P@10 & P@15 \\
\hline 1 & 0.6 & 0.4 & 0.267 & 11 & 0.6 & 0.4 & 0.267 \\
\hline 6 & 1 & 0.9 & 0.667 & 16 & 1 & 0.7 & 0.733 \\
\hline 21 & 0.6 & 0.4 & 0.4 & 30 & 0.2 & 0.3 & 0.333 \\
\hline Average & 0.73 & 0.56 & 0.445 & Average & 0.6 & 0.46 & 0.444 \\
\hline
\end{tabular}

Table 14. Number of unique query terms and average idf in the queries formulated for topic 443.

\begin{tabular}{|l|c|c|c|c|c|}
\hline \multicolumn{3}{|c|}{ Experimental system } & \multicolumn{3}{c|}{ Control system } \\
\hline User & $\begin{array}{c}\text { Unique query } \\
\text { terms excluding } \\
\text { stopwords }\end{array}$ & $\begin{array}{c}\text { Average idf of } \\
\text { non-stopword } \\
\text { query terms }\end{array}$ & User & $\begin{array}{c}\text { Unique query } \\
\text { terms excluding } \\
\text { stopwords }\end{array}$ & $\begin{array}{c}\text { Average idf of } \\
\text { non-stopword } \\
\text { query terms }\end{array}$ \\
\hline 1 & 6 & 6.256 & 11 & 7 & 6.053 \\
\hline 6 & 12 & 4.964 & 16 & 8 & 4.712 \\
\hline 21 & 27 & 5.114 & 30 & 12 & 4.045 \\
\hline Average & 15 & 5.445 & Average & 9 & 4.937 \\
\hline
\end{tabular}

As can be seen from Table 13, the three queries formulated using the experimental interface on average performed better in $\mathrm{P} @ 5$ and $\mathrm{P} @ 10$. Although the average number of unique query terms entered via the templatebased interface is higher than in the control, more words do not always lead to higher performance. As evident from Table 14, users 21 and 6 entered 27 and 12 unique words respectively (with the average idf of 5.114 and 4.964), but the P@15 was 0.4 and 0.667 respectively. Interestingly, there is very little overlap between the top 15 documents retrieved by the six users given topic 443: 74 out of 90 documents were retrieved only once, one document was retrieved by four users, and six documents were retrieved by two users. Only one document was judged inconsistently by two users. 


\section{Olga Vechtomova and Hao Zhang}

\section{Conclusions and future work}

In this paper we proposed an approach to help users articulate complex information needs by filling in topicindependent query templates. A complex information need in our understanding is a need to learn about specific semantic relations between entities or aspects of an entity. By suggesting to a user a list of templates formulated as fill-in-the-blanks questions, our goal is to encourage users to think about and express their information needs in terms of relations between the entities of their interest, such as what causes $X$, what can be done to prevent $X$, what effect does $\mathrm{X}$ have on other entities. We developed an experimental interface with a set of topic-independent query templates and a control interface with a single query textbox, and conducted a user study, which aimed to answer two research questions:

\section{RQ1: Are users able to articulate complex information needs using topic-independent query templates?}

The data gathered through query and search session logs suggests that users are able to express their information needs by filling in query templates. On average the users completed 7.8 templates out of 13 . Also, the average number of distinct query terms excluding stopwords entered using the template-based system is slightly higher than that of the control system.

RQ2: When used with a bag-of-words retrieval system, do the queries formed using template-based interface lead to better search results than queries formed using a single-textbox interface?

The template-based interface helped the users formulate on average more effective queries, resulting in better search results (measured in precision at 5, 10 and 15 documents) compared to the control system. Improvement in P@10 is statistically significant. The average user satisfaction levels with the search results and the overall system reported in the questionnaire are also higher for the template-based system. At the same time, the users' subjective ratings of the easiness of formulating queries indicate that on average they found query formulation by filling in templates not as easy as entering their query into one textbox. However, the average time spent on formulating the query using templates is only 27 seconds longer than using one textbox.

Some of the users found some templates to be too restrictive. Such templates need to be re-written to improve their clarity and applicability to a wider range of queries. For example, a user interested in "measures to combat music piracy" may not think that the template "What has been done (could be done) to alleviate/reduce the effect of on ___ ? ? is appropriate for expressing his information need.

An interesting future research direction may be automatically selecting a subset of templates to show to the user on the basis of his initial short query statement. It would be desirable to tune the templates to a specific query, thus avoiding showing to the user those templates, which are unlikely to be relevant to his need. In this way it would be possible to build a larger set of more detailed templates, with only some of them being shown to the user as fill-in-theblanks clarification questions.

In the present work our goal was to study template-filling as a query formulation method, we did not use the relationships specified by the users in the query templates in document retrieval and ranking. Advances in the research in automatic identification of semantic relations in text, for example done within the framework of RTE (Recognising Textual Entailment) track of TAC (Text Analysis Conference) [11] may facilitate the retrieval of documents, which match the specific relation expressed by the user in the query. The combination of such document retrieval methods with the template-based interfaces may be useful, helping users to express the relationships between entities they are interested in and receive documents that specifically refer to these relationships. 


\section{Olga Vechtomova and Hao Zhang}

\section{References}

[1] S. E. Robertson, S. Walker, and M. M. Hancock-Beaulieu, Experimentation as a way of life: Okapi at TREC. Information Processing and Management 36(1) (1999) 95-108.

[2] N. J Belkin., R. N. Oddy and H. M. Brooks, ASK for Information Retrieval: Part I. Background and Theory. Journal of Documentation, 38(2) (1982) 61-71.

[3] C. Buckley, G. Salton. and J. Allan, The effect of adding relevance information in a relevance feedback environment. In: Proceedings of the 17th ACM-SIGIR, 1994, Dublin, Ireland.

[4] B. J. Jansen, A. Spink and T. Saracevic, Real life, real users and real needs: a study and analysis of user queries on the web. Information Processing and Management, 36(2) (2000) 207-227.

[5] N. J. Belkin, C. Cool, D. Kelly, S. Lin, S. Y. Park, J. Perez-Carballo and C. Sikora, Iterative exploration, design and evaluation of support for query reformulation in interactive information retrieval. Information Processing and Management, 37(3) (2001) 404-434.

[6] D. Kelly, V. J. Dollu and X. Fu, The loquacious user: a document-independent source of terms for query expansion. In: Proceedings of the 28th ACM-SIGIR, 2005, Bahia, Brazil.

[7] L. S. Price, M. Lykke, L. M. L. Delcambre, P. Vedsted, Semantic Components Enhance Retrieval of Domain-Specific Documents. In: Proceedings of CIKM 2007, Lisbon, Portugal.

[8] D. Kelly and J. Lin, Overview of the TREC 2006 ciQA task. SIGIR Forum, 41(1) (2007) 107-116

[9] C. Macdonald, I. Ounis, I. Soboroff, Overview of the TREC 2007 Blog Track.

[10] E. Agirre, L. Marquez and R. Wicentowski (Eds.) Proceedings of the 4th International Workshop on Semantic Evaluations (SemEval 2007), June 23-24, 2007, Prague, Czech Republic.

[11]D. Giampiccolo and H. Trang Dang (track coordinators) TAC 2008 Recognizing Textual Entailment (RTE) Track. Available at: http://www.nist.gov/tac/tracks/2008/rte/ (accessed 25 September 2008)

[12] M. Hearst Automatic acquisition of hyponyms from large text corpora. In: Proceedings of the 14th International Conference on Corpus Linguistics (COLING-92), 1992.

[13] P. Turney and M. Littman Corpus-based learning of analogies and semantic relations. Machine Learning, 60(1-3) (2005) 251-278.

[14] P. Nakov and M. Hearst, UCB: system description for SemEval task \#4. In: Proceedings of the 4th International Workshop on Semantic Evaluations, Prague, June 2007.

[15] B. Beamer, S. Bhat, B. Chee, A. Fister, A. Rozovskaya and R. Girju, UIUC: a knowledge-rich approach to identifying semantic relations between nominals. In: Proceedings of the 4th International Workshop on Semantic Evaluations, Prague, June 2007.

[16] M. Hurst and K. Nigam, Retrieving topical sentiments from online document collections. In: Proceedings of the 11th Conference on Document Recognition and Retrieval, 2004.

[17]J. Yi, T. Nasukawa, R. Bunescu, and W. Niblack, Sentiment analyzer: extracting sentiments about a given topic using natural language processing techniques. In: Proceedings of the 3rd IEEE International Conference on Data Mining, 2003. 


\section{Olga Vechtomova and Hao Zhang}

[18] K. Yang, N. Yu, A. Valerio and H. Zhang, WIDIT in TREC-2006 Blog track. In: E. Voorhees and L. Buckland (eds.) Proceedings of the 15th Text Retrieval Conference (TREC), Gaithersburg, MA., November, 2006.

[19] W. C. Mann and S. A. Thompson, Rhetorical Structure Theory: Toward a functional theory of text organization. Text, 8 (3) (1988) 243-281.

[20] http://www.sfu.ca/rst/01intro/definitions.html (accessed 25 September 2008).

[21] K. Spärck Jones, S. Walker and S.E. Robertson, A probabilistic model of information retrieval: development and comparative experiments. Information Processing and Management, 36(6) (2000) 779-808 (Part 1) and 809-840 (Part 2).

[22] J. Allan, HARD track overview in TREC 2004. High accuracy retrieval from documents. In: E. Voorhees and L. Buckland (eds.), Proceedings of the 13th text retrieval conference, Gaithersburg, MD, USA, November 2004. 